information was used to collect data. Data were analysed in a descriptive fashion.

Results In all 27883 individuals were studied. The mean age of respondents was 32.7 ( $\mathrm{SD}=11.8$ ) years. Overall 25.4\% were smoker, $53.8 \%$ indicated that never or a little did exercise, $52.7 \%$ indicated that they were very or very much concerned about their nutrition; $20.1 \%$ reported high level of anxiety, $25.6 \%$ reported a degree of depression, 30\% stated that they did not care about their health, $79.3 \%$ reported a relatively high level of relationships with family, relatives and friends, $20.7 \%$ reported that they suffer from at least one chronic disease, and $27.9 \%$ reported less than good health status. Only $36.7 \%$ of the respondents said that they have enough information about health.

Conclusion Considering current healthcare programs in Iran it seems that policy makers should have a better understanding of people's health-related attitudes and behaviours.

\section{P1-252 CREUTZFELDT-JAKOB DISEASE (CJD) SURVEILLANCE IN TEXAS2010: A SURVEILLANCE ODYSSEY}

doi:10.1136/jech.2011.142976e.44

\section{K Moody.* Texas Department of State Health Services, Austin, Texas, USA}

Creutzfeldt-Jakob Disease (CJD) is a rare, invariably fatal neurodegenerative disease caused by misfolded proteins called prions. The disease can originate in three different ways (sporadic, genetic and infectious) and has four forms (sporadic, variant, iatrogenic, and familial). Known infectious routes include ingestion of contaminated beef (mad cow disease or vCJD), and certain surgical procedures and contaminated surgical instruments (iCJD). CJD is a difficult disease to diagnose; ultimately requiring neuropathological examination of brain tissue for confirmation. Clinical history, MRI, electroencephalogram and cerebrospinal fluid testing assessing 14-33 and Tau proteins levels are all tools that are used to assess the likelihood of CJD. Unlike most reportable conditions, CJD can only be confirmed after death. Because of the complexity of the disease, surveillance and case investigation can be a challenge. The average rate of CJD in the US is 1 case per million. Historically, Texas has averaged 16 cases per year (0.7 cases per million). Last year, 2010, was an exception. Not only did Texas have over 25 cases, 7 of these cases had an uncharacteristic clinical course. This presentation will take a historical look at CJD surveillance in Texas from 2000 to 2010 and will highlight the distinctiveness of 2010 .

\section{P1-253 LONG-TERM HEALTH RISKS AFTER INFECTIVE GASTROENTERITIS: A POPULATION-BASED COHORT STUDY}

doi:10.1136/jech.2011.142976e.45

\footnotetext{
${ }^{1,2} \mathrm{R}$ Moorin, ${ }^{*} \mathrm{~J}$ Heyworth, ${ }^{3} \mathrm{G}$ Forbes, ${ }^{2} \mathrm{~T}$ Riley. ${ }^{1}$ Curtin University, Western Australia, Australia; ${ }^{2}$ The University of Western Australia, Western Australia, Australia; ${ }^{3}$ Royal Perth Hospital, Western Australia, Australia
}

Introduction Gastroenteritis presents a considerable burden on communities and health systems. In addition to the immediate health consequences long-term adverse sequelae have been associated with gastroenteritis; however, the risk and type of sequelae attributable to previous exposure to enteric infections have not been quantified. The objective of this study was to quantify the risk and type of sequelae attributable to previous exposure to enteric infections occurring in childhood and adolescence.

Methods This was a population-based retrospective cohort study comprising all individuals born in Western Australia between 1 January 1985 and 31 December 2000 using linked administrative records from the Western Australian notifiable infectious diseases database, the Western Australian hospital morbidity data system and Western Australian death notifications. The risk of first-time hospitalisation for sequelae for those exposed to an enteric infection was modelled using Cox proportional regression analysis controlling for other health and socio-demographic factors.

Results After adjusting for confounding there was a significant increase in the rate of first-time hospitalisation for sequelae in those exposed to enteric infections by $64 \%$ for any sequelae; $52 \%$ for intragastrointestinal sequelae and $63 \%$ for extra-gastrointestinal sequelae compared with non-exposed individuals. This equated to an increased attributable risk of 39\% for all sequelae, 34\% for intragastrointestinal and 39\% for extra-gastrointestinal sequelae.

Conclusion Infective gastroenteritis in childhood or adolescence increases the risk of first-time hospitalisation for intra-gastrointestinal and extra-gastrointestinal disease over the following 2 decades. This highlights the importance of developing appropriate risk management strategies for those exposed to enteric infections.

\section{P1-254 DETERMINANTS OF REFUSALS IN A FOLLOW-UP EVALUATION OF A PORTUGUESE BIRTH COHORT}

doi:10.1136/jech.2011.142976e.46

1,2 V Morais, ${ }^{*}{ }^{1,2} \mathrm{~S}$ Correia, ${ }^{1,2} \mathrm{~A}$ C Santos, ${ }^{1,2} \mathrm{H}$ Barros. ${ }^{1}$ Department of Hygiene and Epidemiology, University of Porto Medical School, Porto, Portugal; ${ }^{2}$ Institute of Public Health, University of Porto, Porto, Portugal

Introduction The main advantages of birth cohort studies may be compromised by the refusals in participation, which tend to increase in time.

Objective To describe the main predictors of refusals in the Portuguese birth cohort 4 years after the assembling.

Methods Geração XXI was assembled in 2005/2006 in five public maternities in Porto Metropolitan area, Portugal. After delivery, data were collected by personal interviews. Sub-samples of mothers were re-assessed at 6, 15 and 24 months and an ongoing evaluation of the entire cohort is being performed at the age of $4 / 5$ years. Refusals at follow-up were compared with evaluated participants considering baseline maternal and childhood characteristics, fitting logistic regression models were adjusted for age, education level and working condition, place of residence and previous follow-ups.

Results Within 6025 participants already contacted, 88\% were evaluated and $704(12 \%)$ refused. The major determinant of refusal was the absence of previous evaluations (OR $=2.01 ; 95 \%$ CI 1.68 to 2.41). Refusals were more frequent among less educated women ( $\leq 9$ vs $\geq 13$ years of education: $\mathrm{OR}=1.23$; $95 \% \mathrm{CI} 1.01$ to 1.53 ), as within housewives (vs employed $\mathrm{OR}=1.60 ; 95 \%$ CI 1.14 to 2.25 ). No statistical significant differences were found regarding other maternal, birth or newborn characteristics. The main reasons for refusal were unavailability (39.6\%), personal/professional reasons (18.9\%) and distance to evaluation setting (16.1\%).

Conclusions This analysis suggests that keeping regular contacts with participants is probably the best approach to reduce refusals, which were, in this cohort, mainly determined by maternal sociodemographics characteristics.

\section{P1-255 TRENDS OF BRUCELLOSIS (MALTA FEVER) IN IRAN DURING THE PERIOD 1991-2008}

doi:10.1136/jech.2011.142976e.47

${ }^{1} \mathrm{E}$ Mostafavi, ${ }^{*} \mathrm{M}$ Asmand. ${ }^{1}$ Pasteur Institute of Iran, Tehran, Iran; ${ }^{2}$ Faculty of Veterinary Medicine, University of Tabriz, Tabriz, Iran

Introduction Brucellosis (Malta fever) is a bacterial zoonotic infectious disease. This study reviews the brucellosis trend in Iran during 1991-2008, provides the distribution maps related to the patient 
and assays the effect of livestock vaccination rate on disease report in humans.

Methods Necessary Information was achieved from concerned authorities. Data analysis was done by SPSS software version 16 . Mapping was performed using ArcGIS version 9.3.

Results Brucellosis is reported more in the West and Northwest provinces, where their sheep and goat population density is higher than other parts of the country. The highest incidence of brucellosis was seen in 1991 with a rate 101.63 per one hundred thousand people. On average, each year about 27500 new cases were reported in the country. Brucellosis incidence over the years showed a decreasing trend $(r=-0.79, \mathrm{P}<0.001)$. Pearson correlation analysis showed that in different provinces there was a significant association between the annual incidence of Malta fever and the annual infection rate of sheep and goats $(r=0.347, p<0.001)$, the annual incidence of Malta fever and the annual infection rate of cattle $(\mathrm{r}=0.327, \mathrm{p}<0.001)$ and the annual incidence of Malta fever and the percentage of vaccinated cows $(r=0.098, p=0.031)$.

Conclusion As the prevalence of brucellosis depends very closely with the prevalence of brucellosis in animals, the control of the disease in human population is not possible unless it is controlled in livestock; so the cooperation between different organizations (Veterinary, public health ...) is needed.

\section{P1-256 WHAT NEXT MEASLES CONTROL FOR KARNATAKA INDIA?}

doi:10.1136/jech.2011.142976e.48

R Mugali. ${ }^{*}$ German TB leprosy relief association, Chennai, India

Introduction Worldwide, measles still kills 400 people every day, more than $90 \%$ of them being under-fives. Three out of four deaths happen in India, The objectives of my work are to describe the epidemiology of measles in Karnataka and to identify ways to improve measles control in the state.

Methodology I collated and tabulated weekly surveillance reports and outbreak investigation line lists for 4 years. I synthesised the spatio-temporal distribution of measles cases and outbreaks in the state. Through logistic regression multivariate analysis, specified the contextual demographic and socio-cultural determinants of measles outbreaks and vaccination coverage in the state of Karnataka.

Findings Measles surveillance data from 2006 to 2009 reveal that measles is indeed endemic in Karnataka, with frequent outbreaks. The notification rate of measles is 10.94 cases per 100000 population per year. Seasonality of notified measles cases characteristically increases between November and April and decreases from May to October. There were 163 confirmed outbreaks in the state in the 4 years under study. Measles outbreaks were happening consistently in the northern part of the state and sporadically in the southern part: The duration of these outbreaks from the date of appearance of rash in a first case to last case was ranged from 7 days to 120 days with median of 39 days.

Discussion Significant reduction in measles incidence and outbreaks can be attained only by adding a second dose to the routine vaccination scheme in the whole state and by conducting catch-up campaigns in northern Karnataka.

\section{P1-257 RELATIONSHIP BETWEEN THE NUMBER OF ESTABLISHED CARDIOVASCULAR RISK FACTORS AND ANNUAL MEDICAL COSTS IN JAPAN: COST ANALYSIS IN 38890 JAPANESE INDIVIDUALS}

doi:10.1136/jech.2011.142976e.49

${ }^{1} Y$ Murakami, ${ }^{2} \mathrm{~T}$ Okamura, ${ }^{3} \mathrm{~K}$ Miura, ${ }^{4} \mathrm{H}$ Ueshima. ${ }^{1}$ Department of Medical Statistics, Shiga University of Medical Science, Otsu, Shiga, Japan; ${ }^{2}$ Department of Preventive Medicine and Public Health, Keio University, Shinjuku, Tokyo, Japan; ${ }^{3}$ Department of
Health Science, Shiga University of Medical Science, Otsu, Shiga, Japan; ${ }^{4}$ LifestyleRelated Disease Prevention Center, Shiga University of Medical Science, Otsu, Shiga, Japan

Background Information about the relationship between the number of established cardiovascular disease risk factors and medical costs is limited.

Methods A linked analysis of the medical costs and health examination measures was performed in Shiga, Japan. Medical costs information for the period April 2000-May 2006 was collected from the Shiga National Health Insurance Organization database. Corresponding data for 38890 individuals (age, $\geq 40$ years) generated during annual health examination conducted between 2000 and 2003 were collected from the local municipalities in the Shiga prefecture. These data were merged by name, sex, and dates of birth. Median and interquartile were used to compare the annual medical costs among groups with different number of risk factors. The risk factors were hypertension ( $\mathrm{SBP} \geq 140$ or $\mathrm{DBP} \geq 90$ ), high cholesterol (total cholesterol $\geq 240$ ) and blood glucose levels (casual blood glucose $\geq 126$ ), and smoking.

Results The median total annual medical cost in the group with no risk factors was 217149 Yen for men and 147880 Yen for women. The median total annual medical cost in the group with four risk factors was 1.28 times higher in men (278 651 Yen) and 1.80 times higher in women (265.788 Yen), as compared to that in the group with no risk factors. These trends were most apparent in outpatients (men: 1.47 times (127205 Yen vs 186811 Yen); women: 2.23 times (94648 Yen vs 211123 Yen)).

Conclusion Annual medical costs increase with an increase in the number of cardiovascular disease risk factors in the Japanese population.

\section{P1-258 IS AWARENESS OF DOTS AMONG MEDICAL PRACTITIONERS A WORRY? A DEVELOPING NATION SCENARIO}

doi:10.1136/jech.2011.142976e.50

V Nagaraja, ${ }^{*}$ M A Khan, G B Sankapithilu. Mysore Medical College \& Research Institute, Mysore, Karnataka, India

Background Annually two million people in India develop Tuberculosis and 330000 die. WHO-recommended DOTS strategy was pilot-tested in 1993 and launched as Revised National Tuberculosis Control Programme (RNTCP) in 1997. Awareness of DOTS among the doctors in the private sector was appalling although nationwide coverage was attained by 2006.

Objective To study awareness of DOTS among Medical Practitioners of urban and rural Mysore.

Methodology 401 Medical practitioners in hospitals and nursing homes of urban and rural areas of Mysore who treated Tuberculosis patients (private and public sector) were approached. They were grouped under different specialties as per the year of graduation (before or after introduction of DOTS).

Results Thirty eight per cent doctors who graduated before the introduction of DOTS didn't follow DOTS compared to $14.9 \%$ doctors who graduated later. 100\% doctors working in Government sector felt that DOTS was better than daily regimen while $85 \%$ from the private sector felt so. Only $47.9 \%$ of the doctors in the private sector practiced DOTS compared to $95.1 \%$ in the Govt. Sector. Hence, the number of doctors practicing DOTS in Private Sector was $<50 \%$ of that in the Govt. Sector. Both of these comparisons were found to be statistically highly significant $(p<0.001)$. Awareness of DOTS was alarmingly low among Orthopaedic Surgeons, Gynaecologists and Paediatricians when compared to Physicians and General Practitioners. 